\title{
Mucus penetrating properties of soft, distensible lipid nanocapsules
}

Article

Accepted Version

Creative Commons: Attribution-Noncommercial-No Derivative Works 4.0

Chen, H., Mansfield, E. D. H., Woods, A., Khutoryanskiy, V. V., Forbes, B. and Jones, S. A. (2019) Mucus penetrating properties of soft, distensible lipid nanocapsules. European Journal of Pharmaceutics and Biopharmaceutics, 139. pp. 7684. ISSN 0939-6411 doi: https://doi.org/10.1016/j.ejpb.2019.02.020 Available at https://centaur.reading.ac.uk/82562/

It is advisable to refer to the publisher's version if you intend to cite from the work. See Guidance on citing.

To link to this article DOI: http://dx.doi.org/10.1016/j.ejpb.2019.02.020

Publisher: Elsevier

All outputs in CentAUR are protected by Intellectual Property Rights law, including copyright law. Copyright and IPR is retained by the creators or other copyright holders. Terms and conditions for use of this material are defined in the End User Agreement.

\section{www.reading.ac.uk/centaur}

\section{CentAUR}


Central Archive at the University of Reading

Reading's research outputs online 


\section{Accepted Manuscript}

Research paper

zurepean

lournal of

Pharmaceutuies and

Biopharmacentics

Mucus penetrating properties of soft, distensible lipid nanocapsules

Hanpeng Chen, Edward D.H. Mansfield, Arcadia Woods, Vitaliy V.

Khutoryanskiy, Ben Forbes, Stuart A. Jones

PII:

S0939-6411(18)30791-4

DOI:

https://doi.org/10.1016/j.ejpb.2019.02.020

Reference:

EJPB 12995

To appear in: $\quad$ European Journal of Pharmaceutics and Biophar-

maceutics

Received Date: $\quad 21$ June 2018

Revised Date: $\quad 24$ December 2018

Accepted Date: $\quad 23$ February 2019

Please cite this article as: H. Chen, E.D.H. Mansfield, A. Woods, V.V. Khutoryanskiy, B. Forbes, S.A. Jones, Mucus penetrating properties of soft, distensible lipid nanocapsules, European Journal of Pharmaceutics and Biopharmaceutics (2019), doi: https://doi.org/10.1016/j.ejpb.2019.02.020

This is a PDF file of an unedited manuscript that has been accepted for publication. As a service to our customers we are providing this early version of the manuscript. The manuscript will undergo copyediting, typesetting, and review of the resulting proof before it is published in its final form. Please note that during the production process errors may be discovered which could affect the content, and all legal disclaimers that apply to the journal pertain. 


\section{Mucus penetrating properties of soft, distensible lipid nanocapsules}

Hanpeng Chen ${ }^{\mathrm{a}}$, Edward. D. H. Mansfield ${ }^{\mathrm{b}}$, Arcadia Woods ${ }^{\mathrm{a}}$, Vitaliy. V. Khutoryanskiy ${ }^{\mathrm{b}}$, Ben Forbes $^{\mathrm{a}}$, Stuart. A. Jones ${ }^{\mathrm{a}, *}$

${ }^{\text {a }}$ Institute of Pharmaceutical Science, Franklin-Wilkins Building, King's College London, 150 Stamford Street, London SE1 9NH, UK.

${ }^{\mathrm{b}}$ Reading School of Pharmacy, University of Reading, Whiteknights, P.O. Box 224, Reading, Berkshire RG6 6AD, UK

*Corresponding author. Dr. S. A. Jones. King's College London, Faculty of Life Sciences \& Medicine, Institute of Pharmaceutical Science, Franklin-Wilkins Building, 150 Stamford Street, London, SE1 9NH. Tel: +44 (0)207 848 4843. Fax: +44 (0)207 848 4800. Email:stuart.jones@kcl.ac.uk 


\section{ABSTRACT}

Designing nanomaterials to release their drug pay-load upon exposure to an exogenous trigger can help to direct drug delivery, but how the triggered release, which often modifies the nanomaterial properties, influences the biological fate of these systems is currently unknown. The aim of this study was to investigate how the triggered drug release from PEG coated, soft, $50 \mathrm{~nm}$ distensible lipid nanocapsules (LNC) influenced their diffusion across a mucus barrier. The translocation speed of the non-triggered LNC across a $35 \mu \mathrm{m}$ thick purified gastric mucin $(\mathrm{PGM})$ barrier was 3 times faster $\left(30.08 \pm 2.49 \times 10^{-10} \mathrm{~cm}^{2} \mathrm{~s}^{-1}\right)$ compared to equivalent-sized negatively charged polystyrene particles $\left(9.87 \pm 0.61 \times 10^{-10}\right.$ $\left.\mathrm{cm}^{2} \mathrm{~s}^{-1}, \mathrm{p}<0.05\right)$. In cystic fibrosis mucus $(\mathrm{CFM})$, harvested from patient primary cells, the non-triggered LNC translocation speed was similar to the PGM, but the polystyrene particles diffusion was so slow it could not be measured. The trigger induced LNC distension process had no effect on the particle diffusion rate in both PGM and CFM ( $p>0.05)$ in a static mucus barrier, but when shear was applied to the barrier the distended LNCs diffused more slowly $\left(3.97 \pm 1.38 \times 10-8 \mathrm{~cm}^{2} \mathrm{~s}^{-1}, \mathrm{p}<0.05\right)$ compared to the non-distended materials $(4.94 \pm 0.04 \mathrm{x}$ $10-8 \mathrm{~cm}^{2} \mathrm{~s}^{-1}$ ). This data suggested the rapid mucus penetration of the distended LNCs, despite their increased size, was a consequence of their capacity to take a less tortious path through the barrier, i.e., they experienced less steric hinderance, compared to the non-distended LNC.

Key words: mucus; nanoparticle; nanomaterial; diffusion; mucoadhesion; penetration; cystic fibrosis; size, zeta potential, surface charge, polystyrene, lipid. 


\section{INTRODUCTION}

'Soft' nanomaterials can be designed such that they are responsive to a drug release trigger signals. ${ }^{1-3}$ This approach can be particularly advantageous when attempting to deliver sensitive drug payloads, e.g. SiRNA, into epithelial cells, e.g., during the treatment of cystic fibrosis, because it can provide protection from enzymatic processes prior to efficient cellular uptake. ${ }^{4-6}$ However, to deliver therapeutic agents into epithelial cells using trigger sensitive nanomaterials they need to translocate across a mucus barrier effectively and their ability to do this is currently unknown.

Previous work has demonstrated that non-trigger responsive nanomaterials can diffuse through cystic fibrosis (CF) sputum, ${ }^{7}$ human respiratory mucus, ${ }^{8}$ human buccal mucosa, ${ }^{9}$ human rhinosinusitis mucus, ${ }^{10}$ porcine gastric mucin $(\mathrm{PGM})^{11}$ and mucus expressed by cell lines $^{12}$. However, the diffusion of trigger sensitive nanomaterials through mucus could be impeded as a consequence of the nanomaterial changes induced by the triggering system. It is not easy to predict how the triggered drug release will influence mucus diffusion because a relationship between nanomaterial transport through mucus and particle characteristics has yet to be clearly defined.

Nanomaterial diffusion through gels is hindered by two physical phenomena, hydrodynamic and steric interactions ${ }^{17,18}$ (Equation 1):

$$
\frac{D_{e f f}}{D_{0}}=F S\left(d_{f}, \phi\right)
$$

where $D_{\text {eff }}$ is the diffusion coefficient through the mucus gel, $D_{0}$ is the diffusion coefficient through water, $F$ is the hydrodynamic interactions and $S\left(d_{f}, \phi\right)$ the steric 
interactions (both $\mathrm{F}$ and $\mathrm{S}$ range from $0-1$ ). The steric interactions are a function of the size exclusion process imparted by the gel and the hydrodynamic interactions are a function of the frictional retardation that is imparted on the nanomaterial diffusion, i.e., the nanomaterial gel interactions. However, because nanomaterial diffusion through mucus does not show Stokesian dynamics (mucus is a non-Newtonian gel) and it is difficult to use micro-rheology techniques to understand the interactions between nanomaterials and mucus, a validated approach to isolate and calculate both steric and hydrodynamic interactions of drug delivery nanomaterials with mucus does not currently exist ${ }^{14-16}$. As a consequence, nanomaterial mucus barrier interactions are often studied using combinations analytical approaches to probe the effects of nanomaterial characteristics on mucus diffusion. For example, particle tracking, fluorescence correlation spectroscopy, dynamic light scattering and pulsed-field gradient NMR. ${ }^{16}$ However, these approaches have yet to be applied to soft trigger responsive nanomaterials in order to understand their diffusion in mucus barriers.

The aim of this study was to investigate how soft nanomaterials that were responsive to drug release trigger signals interacted with and diffused through mucus. Lipid nanocapsules (LNCs) were selected as the soft, mucus penetrating nanomaterials for this work because, in addition to their PEG decorated surface, which has previously been shown to assist diffusion through mucus, ${ }^{14}$ they were amenable to trigger responsive drug delivery through a particle distension process (size increase from $50 \mathrm{~nm}$ to $200 \mathrm{~nm}$ ) upon exposure to a Pluronic surfactant (Fig 1). ${ }^{1,19}$ It was envisaged that in a final presentation of the inhaled formulation the trigger would be mixed with the nanoparticles upon dose actuation as this would allow nanoparticle distension to occur after deposition upon the airway epithelia. In order to aid the understanding of how the LNCs interacted with the mucus they were compared with nontrigger sensitive polystyrene nanoparticles with diameters of 50, 200 and $750 \mathrm{~nm}$. The 
particle size range of the polystyrene materials was selected to cover the distensible size of the LNCs and the typical sizes of materials used for mucosal administration of therapeutic agents. The $200 \mathrm{~nm}$ polystyrene particles were chosen to investigate the types of interactions that hindered particle diffusion through varying the $\mathrm{pH}$ of the mucus. In addition, in order to understand the manner in which the mucus properties influenced the interactions with the nanomaterials diffusion studies were conducted in both PGM and human cystic fibrosis mucus (CFM), derived from human primary airway epithelial cells donated from a homozygous CF donor ${ }^{20,21}$. These two types of mucus were specifically chosen due to the difference in extracellular material in the two barriers, the PGM was purified to remove the extracellular component and thus display comparatively less steric interactions with the nanomaterials compared with the CFM, which was used as recovered from the cells and thus contained a higher concentration of extracellular material. A static Transwell was used to measure particle diffusion speed during translocation across the mucus via a chemical assay. The particle diffusion speed in Transwell assay was considered to be influenced by both steric and hydrodynamic particle interactions. In contrast, a second assay which measured particle diffusion using multi-particle tracking employed shear to disrupt the mucus structure, this diminished the mucus's capacity to hinder diffusion through steric interactions with the nanomaterials and thus render the hydrodynamic interactions more consequential when considering particle diffusion speed in these measurements. ${ }^{13}$ 


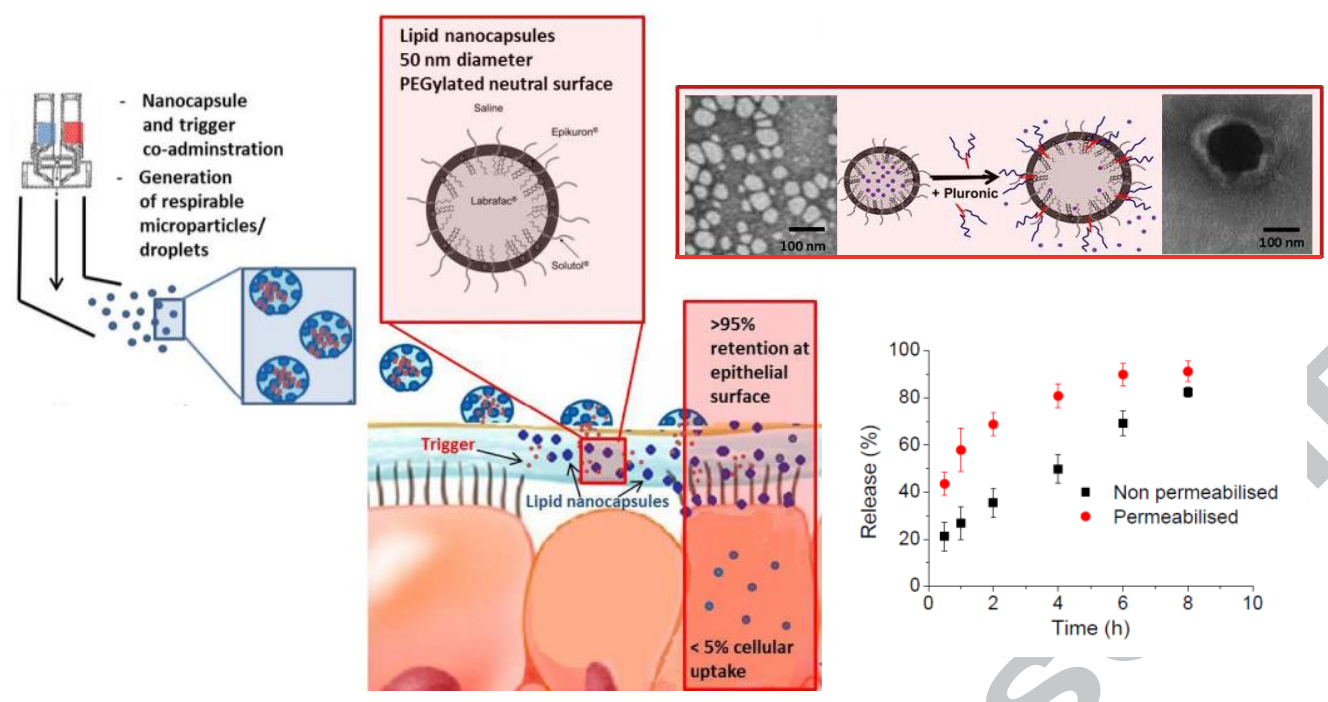

Fig 1. Illustration of the delivery, absorption and drug release of the lipid nanocapsules that distended in response to co-administration with a Pluronic 'trigger'. Images adapted from Chen et al, 2016 ${ }^{1}$ and Chana et al, $2015^{19}$.

\section{MATERIALS AND METHODS}

\subsection{Materials}

Pig stomachs were purchased from Mutch Meats (Whitney, UK). The CFM was from Epithelix (Epithelix Sarl, Geneva, Switzerland). Fluoresbrite YG carboxylate polystyrene microspheres $(0.05,0.2$ and $0.75 \mu \mathrm{m}, 2.5 \% \mathrm{w} / \mathrm{v})$ were sourced from Polysciences (Eppelheim, Germany). Medium chain triglycerides (Labrafac ${ }^{\circledR}$ lipophile 1349) and Lipoid ${ }^{\circledR}$ S75-3 were kindly supplied by Gattefossé S.A. (Saint-Preist, France) and Cargill GmbH (Germany), respectively. PEG 15 hydroxystearate $\left(\right.$ Solutol $^{\circledR}$ HS15) and Pluronic ${ }^{\circledR}$ surfactant L62D were from BASF (Ludwigshafen, Germany). 1,2-dimyristoyl-sn-glycero-3-phosphoethanolamineN-diethylenetriaminepentaacetic acid (DMPE-DTPA) was purchased from Avanti Polar Lipids Inc, Alabama, USA. Sodium chloride, ethylene diamine tetra acetic acid (EDTA), phenylmethylsulfonyl fluoride (PMSF), sodium azide, N-methyl-2-pyrrolidone, sodium fluorescein salt, Nile red, sodium phosphate dibasic heptahydrate, sodium phosphate 
monobasic monohydrate, thioglycolic acid, trypsin, lysozyme, bovine serum albumin (BSA), transferrin and haemoglobin were purchased from Sigma-Aldrich (Dorset, UK).

\subsection{Mucus Preparation and Characterization}

The porcine mucus collected from the stomachs of freshly slaughtered pigs was purified to remove particulate debris and increase the mucin content. The stomachs were opened along their greater curvature, inverted and any food content removed mechanically. They were rinsed with double-distilled water, the mucus lining was gently removed by scraping and the recovered mucus was mixed in a 1:1 ratio with a protease inhibitor buffer containing $200 \mathrm{mM}$ sodium chloride, $0.02 \%$ (w/v) sodium azide, $5 \mathrm{mM}$ EDTA and $1 \mathrm{mM}$ PMSF. The protease inhibitors prevent the mucin degradation. ${ }^{44}$ The mucus mixture was centrifuged to remove particulate debris at $11,200 \mathrm{~g}$ for $45 \mathrm{~min}$ at $4^{\circ} \mathrm{C}$. The supernatant was poured into Visking dialysis tubing (MWCO: 12-14 kDa, Fisher Scientific, Loughborough, UK) and it was dialysed against at least $10 \mathrm{~L}$ of deionised water for $24 \mathrm{~h}$ to remove the small molecular weight material. The dialysed mucus solution was concentrated using an Amicon ultra-filtration cell (Model 8400, $10 \mathrm{kDa}$ membrane, Merck Millipore, UK) under nitrogen at a pressure of $40 \mathrm{psi}$ and temperature of $4^{\circ} \mathrm{C}$. The retained mucin sample was frozen at $-20^{\circ} \mathrm{C}$ until used. A total of 10 stomachs produced $\sim 16 \mathrm{~g}$ of PGM in the form of a homogenous viscous gel. The dry weight of the purified mucin was determined to be $28 \%$ w/w. Where possible the PGM was used as prepared, i.e., without dilution, as preliminary data showed that this purified mucin had similar rheological properties to non-purified mucus. A single batch of PGM was prepared for all the nanoparticle diffusion studies. 
The elastic $\left(G^{\prime}\right)$ and viscous modulus $\left(G^{\prime \prime}\right)$ of the collected mucin was determined using a $2 \mathrm{~g}$ sample on the Carri-Med rheometer (TA Instruments, US) at a constant stress of 1.8 $\mathrm{Pa}$ (shown not to break down the mucus structure).

The purified mucin swelling was quantified by applying $1 \mu \mathrm{L}$ of the mucin sample onto the apical surface of a Transwell diffusion cell $\left(0.3 \mathrm{~cm}^{2}\right.$ polyester, $3 \mu \mathrm{m}$ pore size, Corning, UK) incubated at $37^{\circ} \mathrm{C}$ using a calibrated positive displacement pipette. Then $600 \mu \mathrm{L}$ of Tris buffer $(50 \mathrm{mM}, \mathrm{pH} 8.5)$ was added to fill the basolateral chamber of the Transwell. The experiment was initiated with the addition of 1,5 or $10 \mu \mathrm{L}$ of the polystyrene nanosuspension on top of the mucin in the apical chamber. At regular time points the change in weight of the mucin barrier was characterised gravimetrically and $50 \mu \mathrm{L}$ samples were removed from the receiver chamber to determine the particle translocation. The sample volume was replaced after each time point to keep the receiver volume constant in the experiments. The amount of fluorescein in the samples removed from the receiver chamber was determined by measuring fluorescence intensity using an excitation of $460 \mathrm{~nm}$ and an emission of $515 \mathrm{~nm}$ at $37^{\circ} \mathrm{C}$ (FLx800 Microplate Fluorescence Reader, Bio-TEK Instruments, UK).

To characterize the molecular weight of the collected PGM and CFM samples, a size exclusion assay was employed. A calibration plot of molecular weight against retention time was obtained for the standards lysozyme, trypsin, peroxidase, BSA and thyroglobulin using the Ominsec software (Malvern Instruments, Worcestershire, UK). The calibration and test samples $(100 \mu \mathrm{L}, 1 \mathrm{mg} / \mathrm{mL}$ ) were injected into a size exclusion column (TSK Gel $3000 \mathrm{SW}$, Tosoh Bioscience LLC, Japan) at a flow rate of $1 \mathrm{~mL} / \mathrm{min}$ using gel permeation chromatography machine equipped with a refractive index detector (Malvern Instruments, Worcestershire, UK). Phosphate buffer $(50 \mathrm{mM})$ with $0.3 \mathrm{M}$ sodium chloride at $\mathrm{pH} 7$ was used as the mobile phase. Both the PGM and CFM mucus samples were provided as frozen 
mucus samples, which were defrosted and directly injected into the size exclusion system (Malvern Instruments, Worcestershire, UK).

\subsection{Nanomaterial Preparation}

The LNCs were manufactured via precipitation from a stable emulsion following repeated phase inversion, as previously described by Heurtault et al. ${ }^{22}$ The emulsion contained medium chain triglycerides $(17 \% \mathrm{w} / \mathrm{w})$, phosphatidylcholine $(1.75 \% \mathrm{w} / \mathrm{w})$, PEG hydroxystearate $(17 \% \mathrm{w} / \mathrm{w})$ and a $3 \% \mathrm{w} / \mathrm{v}$ sodium chloride aqueous solution $(64.25 \% \mathrm{w} / \mathrm{w})$. The LNC suspensions were purified from excess excipients and larger particulate matter via centrifugation (Beckman L8-80 ultracentrifuge, Beckman Coulter, Buckinghamshire, UK), $110,000 \mathrm{~g}$ at $25^{\circ} \mathrm{C}$ for $1 \mathrm{~h}$. The PEG is an integral part of the soft nanocapsule shell and though presents a highly stable nanomaterial. ${ }^{22}$ For the particle tracking analysis, Nile red was loaded into LNCs by dissolving $5 \mathrm{mg}$ of Nile red in $5 \mathrm{~mL}$ acetone and then mixing with the oil phase. The LNC fabrication was then repeated, following the evaporation of acetone, to generate nanocapsules loaded with Nile red. The loading, recovery and retention of the dye in the LNCs has been previously described. ${ }^{19}$

To allow quantification of the LNCs in the mucus translocation studies a radiolabel chelator, $\quad 0.1 \% \quad$ w/w $\quad$ 1,2-dimyristoyl-sn-glycero-3-phosphoethanolamine $\quad$-Ndiethylenetriaminepentaacetic acid (DMPE-DTPA), was incorporated into the shell of the LNC systems. The radiolabel was added to the LNC components during the emulsification step of the nanocapsule manufacture process. This was achieved by diluting the nanosupension to $12 \mathrm{mg} / \mathrm{mL}$ with $0.1 \mathrm{M}$ ammonium acetate buffer (pH 6.6). Indium-111 chloride (Mallinckrodt Medical Inc, Petten, The Netherlands), $\sim 50 \mathrm{MBq}\left({ }^{111} \mathrm{InC}_{13}\right.$, half-life 2.83 days), was dissolved in $0.5 \mathrm{M}$ ammonium acetate ( $\mathrm{pH}$ 5.0) and mixed with LNC50- 
DMPE-DTPA at a ratio of $1: 2 \mathrm{v} / \mathrm{v}$. The mixture was incubated at $37^{\circ} \mathrm{C}$ for $45 \mathrm{~min}$ under gentle shaking. Radiolabelling efficiency and stability was measured by quantifying the radioactivity in the supernatant of the washing solution and washed particle residue after three cycles of washing using spin filtration with Amicon ultrafiltration centrifuge tubes (30 kDa MWCO; Millipore Ltd, Hertfordshire, UK).

\subsection{Nanomaterial Characterization}

The size of the LNCs and the polystyrene nanoparticles were analysed by dynamic light scattering using a Zetasizer Nano ZS (Malvern, Worcestershire, UK). The nanosystems (250 $\mu \mathrm{g} / \mathrm{mL}$ ) were suspended in $50 \mathrm{mM}$ Tris buffer solution $(50 \mathrm{mM}, \mathrm{pH} 8.5)$ and sizes were measured at $37^{\circ} \mathrm{C}$. Tris was used as the electrolyte as, unlike phosphate, it is not known to screen electrolyte interactions in mucus barriers. All measurements were carried out at a scattering angle of $173^{\circ}$ using water as the dispersant. Each measurement comprised of 10 to 14 runs and was performed in triplicate for each sample. Zeta potential measurements were performed at $37^{\circ} \mathrm{C}$ and the nanosuspensions were diluted in Tris buffer $(50 \mathrm{mM}, \mathrm{pH} 8.5)$ to a final concentration of $250 \mu \mathrm{g} / \mathrm{mL}$. Each measurement collected data from between 50 to 100 runs and the measurements were performed in triplicate.

\subsection{Nanomaterial Translocation Studies}

In the translocation experiments the polystyrene diffusion coefficients in undiluted PGM were calculated using a fluorescence assay, whilst the diffusion of the LNCs was calculated using a radiochemical assay. The translocation studies used a Transwell system $\left(0.3 \mathrm{~cm}^{2}\right.$ polyester, $3 \mu \mathrm{m}$ pore size, Corning, UK) was used to support a PGM/CFM layer, which was inserted above a receiver chamber containing $600 \mu \mathrm{L}$ of Tris buffer $(50 \mathrm{mM}, \mathrm{pH}$ 8.5). The particle translocation through the Transwell support was measured in preliminary 
studies, but it was extremely rapid, presumably due to the limited barrier properties of the support alone with contained $3 \mu \mathrm{m}$ holes, and hence a diffusion rate could not be calculated. Therefore, the translocation of sodium fluorescein through barriers with a theoretical PGM thickness of $35,180,350,500,700,1400,2100$ and $2800 \mu \mathrm{m}$ was used to establish the translocation model. The thickness of PGM layers were confirmed by microscopy (Leica CM3050, Leica Microsystems, UK). For the fluorescein translocation experiments, the PGM was placed in the Transwell at $37^{\circ} \mathrm{C}$ for $1 \mathrm{~h}$ to equilibrate before a donor solution containing $100 \mu \mathrm{L}$ of sodium fluorescein $(100 \mu \mathrm{g} / \mathrm{mL})$ in Tris buffer $(50 \mathrm{mM}, \mathrm{pH} 8.5)$ was applied onto the surface of the PGM layer, $t=0$. At regular time points over $30 \mathrm{~h}, 50 \mu \mathrm{L}$ samples were removed from the receiver chamber, an equivalent volume of Tris buffer was replaced into the chamber and the amount of fluorescein in the samples was determined by measuring fluorescence intensity using an excitation of $460 \mathrm{~nm}$ and an emission of $515 \mathrm{~nm}$ at $37^{\circ} \mathrm{C}$ (FLx800 Microplate Fluorescence Reader, Bio-TEK Instruments, UK). The fluorescein assay methodology was shown to be fit for purpose in terms of precision and limit of detection in previous work. ${ }^{19}$ The cumulative mass of sodium fluorescein transferred to the receiver chamber per $\mathrm{cm}^{2}$ of PGM area was calculated, plotted against the time and the PGM barrier translocation rate was calculated from the linear portion of the mass transported vs time plot.

Upon establishing the optimal thickness of barrier to use for the PGM layer, the penetration of the nanoparticles across the Transwell support was tested at $37^{\circ} \mathrm{C}$, the effect of using different donor solution volumes $(1,5$ or $10 \mu \mathrm{L})$ was evaluated using a $35 \mu \mathrm{m}$ thick PGM barrier, the recovery from the experiments was confirmed and the effect of barrier thickness effects on the transport of $1 \mu \mathrm{L}$ of $10 \mathrm{mg} / \mathrm{mL}$ polystyrene nanoparticles was examined. The optimised methodology was used to test the translocation of polystyrene nanoparticles of three different sizes: $50 \mathrm{~nm}, 200 \mathrm{~nm}$ and $750 \mathrm{~nm}$ across both PGM and CFM. 
A particle loading dose of $0.01 \mathrm{mg}$ (i.e. $1 \mu \mathrm{L}$ of a $10 \mathrm{mg} / \mathrm{mL}$ suspension) was applied onto the surface of the PGM layer. The effect of $\mathrm{pH}$ on the transport of $200 \mathrm{~nm}$ polystyrene nanoparticles across both PGM and CFM was studied by adjusting the Tris buffer to $\mathrm{pH} 2.5$, 6.5 and 8.5. Nanoparticle transport was quantified by measuring fluorescence intensity in the receiver fluid as described above and the \% transport was calculated in relation to the applied dose of nanomaterial. The particle translocation rate was converted into a particle diffusion rate (Equation 2):

$$
\frac{d M}{d t}=\frac{D C}{h}
$$

where $\mathrm{dM} / \mathrm{dt}$ was the flux, D was the diffusion coefficient, $\mathrm{C}$ was the concentration of the permeant in the donor solution and $\mathrm{h}$ was the thickness of the barrier. This version of Fick's law is probably the most commonly used in the field of pharmaceutics. It does assume that the concentration gradient across the barrier is linear and constant, i.e. time independent. It is also assumed that the drug substance concentration in the donor compartment is constant and that the concentration in the receiver chamber is virtually zero as compared to the donor concentration. The concentration gradient thus becomes equal to the concentration of the drug substance in the donor chamber at time zero. These assumptions were considered reasonable in all the experiments reported in this work.

In order to verify that particles were present in the receiver fluid at the basolateral side of the PGM barrier, selected samples were analysed using Transmission Electron Microscopy (TEM). These $3 \mu \mathrm{L}$ samples were placed onto a Formvar coated grid, washed for 2 min with deionized water three times and stained with $1 \%$ aqueous uranyl acetate for 3 min at $4{ }^{\circ} \mathrm{C}$ 
until dry. Images were obtained using a FEI Tecnai G2 transmission electron microscope operated at $200 \mathrm{kV}$ fitted with a Gatan Ultrascan US1000 $(2 \mathrm{k} \times 2 \mathrm{k})$ camera.

LNC transport was measured using the same translocation protocol as the polystyrene nanoparticles using both the PGM and CFM barriers. To induce the LNC to distend they were mixed with Pluronic ${ }^{\circledR}$ L62D $(80 \mathrm{mg} / \mathrm{mL})$ to obtain a LNC: surfactant ratio of 1:0.5 w/w. Upon initiating the distension process the particles were immediately applied to the PGM/CFM layer. At appropriate intervals, during the $8 \mathrm{~h}$ diffusion experiment, samples from the Transwell receiver fluid were removed and the radiolabelled LNCs were quantified by scintillation counting. The sample volume was replaced to maintain $600 \mu \mathrm{L}$ in the receiver chamber of the Transwell chamber.

\subsection{Nanomaterial Tracking Studies}

The diffusion of the nanoparticles in a mobile PGM bed was measured directly using a NanoSight LM10 (Malvern Instruments, Worcestershire, UK) with an LM14 top-plate, equipped with a green $532 \mathrm{~nm}$ laser and syringe pump using a method that had previously been shown to be fit-for-purpose with non-Newtonian polymer gels. ${ }^{13}$ All the test samples were diluted by a factor of 100 (i.e. to $0.28 \% \mathrm{w} / \mathrm{w}$ ) and mixed before being injected into the system. It should be noted that the dilution of PGM should not influence the hydrodynamic interactions of the nanomaterials in the gel, which are concentration-independent at the concentration ranges used in this study. ${ }^{23}$ An aliquot of $1 \mathrm{~mL}$ was injected into the NanoSight LM10 and the images were captured with a $560 \mathrm{~nm}$ wavelength filter in place. Then $6 \times 60 \mathrm{~s}$ videos were recorded for each experiment, whilst samples were pushed continuously through the top-plate at a speed of $50 \mathrm{AU}$. This was repeated 3 times, for 3 independent dispersions of particles in the PGM gel. All measurements were conducted at $37^{\circ} \mathrm{C}$. The diffusion 
coefficients were determined for each set of videos using NTA v3.1 software (Malvern, UK) and presented as the mean diffusion coefficient distributions $(n=3) .{ }^{13}$ Using this method a high proportion of particles have previously been shown to diffuse normally and thus the effects of subdiffusion was not quantified in this work. ${ }^{24}$

\subsection{Data and Statistical Analysis}

The particle diffusion in water (Dw) was calculated using the Stokes-Einstein equation assuming a temperature of $37^{\circ} \mathrm{C}$, which was equivalent to the experimental studies. The Dw was used to calculate the $\mathrm{Dm} / \mathrm{Dw}$, which allowed comparison to previous work, e.g., Lai et al., 2011. ${ }^{11}$ SPSS version 20 (IBM, UK) was used for all statistical analyses. The transport data were tested for the normality, the normally distributed data was analysed statistically using one way analysis of variance (ANOVA) and the non-normally distributed data was tested using a non-parametric Kruskal-Wallis test. Post hoc comparisons of the means of individual groups were performed when appropriate using Dunnet's test for normal distributed data and Games Howell test for non-Gaussian distributed data. For all pair-wise comparison of means, Student's independent T-test or Mann-Whitney test was applied.

Differences were considered to be statistically significant at a level of $\mathrm{P}<0.05$.

\section{Results}

\subsection{Purified porcine gastric mucin and cystic fibrosis mucus characteristics}

Size exclusion chromatography demonstrated that the PGM had not undergone significant proteolysis during the preparation stages; molecular weight range of 14 to 860 kDa with an average of $250 \mathrm{kDa}$ (see supplementary data; Fig.S1). The undiluted PGM (28\% w/w) possessed viscoelastic properties with a G' (elastic modulus) that was consistently higher than the G" (viscous modulus), which is typical for mucin containing gels (see 
supplementary data; Fig.S2). ${ }^{25}$ The gel's G' (elastic modulus) was $100.3 \mathrm{~Pa}$ and G', (viscous modulus) was $57.5 \mathrm{~Pa}$ at $10 \mathrm{~Hz}$. The PGM G' was approximately 5 times higher and the G', approximately 15 times higher than equivalent values reported for mucus taken directly from cystic fibrosis patients, ${ }^{26}$ but the sum of these indices $\left(G^{*}\right)$ was about 3 times lower than canine respiratory mucus. ${ }^{27}$ The variance in PGM rheological properties by less than a factor of 10 compared to mucus samples directly taken from in vivo sources substantiated its direct use, i.e., undiluted, in the translocation studies. ${ }^{4}$ The rheology of the CFM was not tested in the current study, due to the its limited availability, but its viscosity was assumed to be within the typical range quoted in the literature. ${ }^{4}$ The CFM displayed an average molecular weight of $412 \mathrm{kDa}$ (see supplementary data; Fig.S3), which was higher than the PGM and consistent with the suggestion that the CFM contained longer oligosaccharides compared to other mucus types. $^{28,29}$ The CFM displayed a higher molecular weight polydispersity compared to the PGM, which was typical for non-purified CFM samples as they contained a high proportion of extracellular material.

\subsection{Nanomaterial Translocation Assay Development}

As the PGM barrier increased in thickness the permeation rate of the fluorescein marker reduced (see supporting information; Fig.S4) and the amount of fluorescent material leaking from the PGM barrier increased (data not shown). Prior to adding the nanomaterials to the PGM barrier in the Transwell, the diffusion of the nanomaterials across the Transwell support was established to be too rapid to measure (data not shown) and the chemical assay recovery was determined $(95 \%)$. When the nanomaterials were added to the apical surface of the PGM using increasing amounts of Tris buffer (total buffer applied increased from $0.05 \mu$ mol to 0.5 $\mu \mathrm{mol}$ ) the $200 \mathrm{~nm}$ polystyrene nanoparticle diffusion was suppressed (see supporting information; Fig.S5), but this did not modify the barrier swelling of the PGM (see supporting 
information; Fig.S5). As a consequence of this preliminary work the subsequent nanomaterial translocation studies used a $1 \mu \mathrm{L}$ sample application volume and a $35 \mu \mathrm{m}$ thick barrier (see supporting information; Fig.S4) because these conditions produced a confluent PGM barrier whilst avoiding significant leaching of mucin fragments into the receiver fluid, which produced fluorescence noise (Fig.2). In addition, the selected nanomaterial translocation experimental conditions allowed a measurable amount of nanoparticles to pass the barrier (Fig.2, TEM images) whilst the barrier retained a thickness that was similar to the respiratory mucus in vivo. ${ }^{30}$
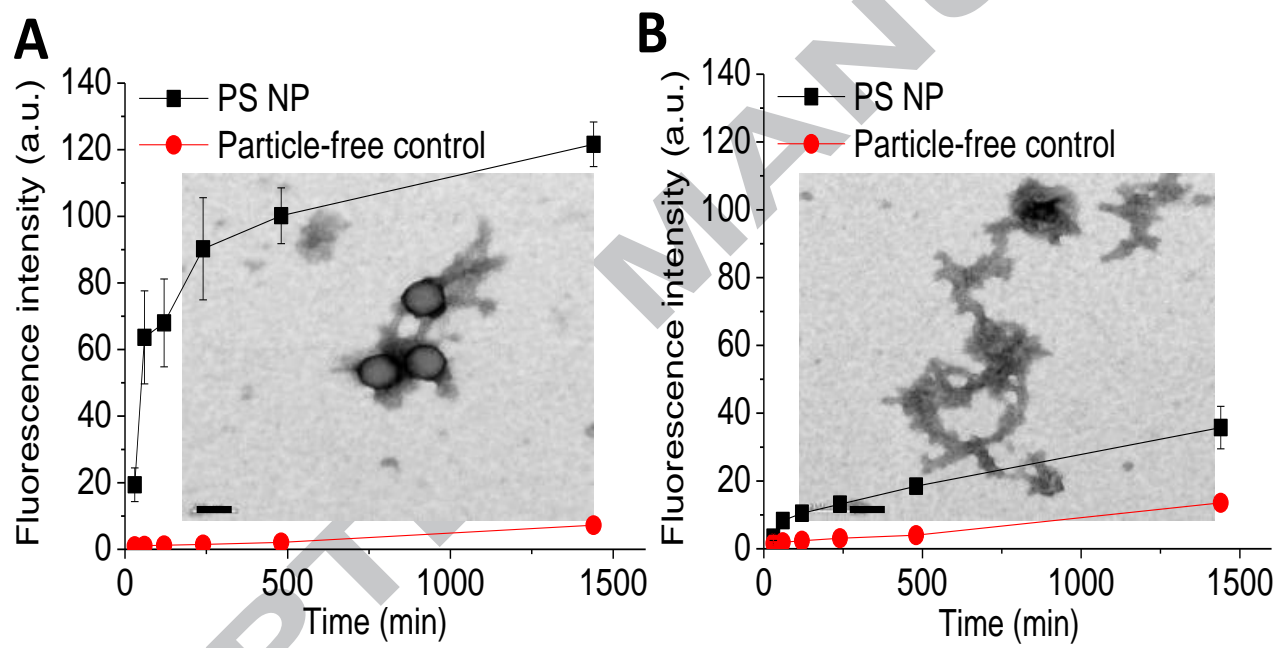

Fig.2.Fluorescence intensity and transmission electron micrograph (inset) of the material in the Transwell receiver chamber following the application of polystyrene $200 \mathrm{~nm}$ nanoparticles or particle-free control (Tris buffer $\mathrm{pH} 8.5$ ) to the purified porcine gastric mucin layers with a thickness of (A) $35 \mu \mathrm{m}$, and (B) $2800 \mu \mathrm{m}$. Data represent mean \pm standard deviation $(\mathrm{n}=3)$. The black scale bar in the images represents $200 \mathrm{~nm}$.

In the initial PGM diffusion experiments the nanomaterial translocation was measured over $24 \mathrm{~h}$. The transport profile from these measurements implied that the particle transport of the polystyrene materials was unidirectional. The subsequent PGM diffusion comparisons were made over $60 \mathrm{~min}$ as this was thought to be more relevant to the typical resonance of nanomaterials in mucus barriers, which are continually replenished in vivo. ${ }^{4}$ 


\subsection{Nanomaterial Translocation across PGM}

The carboxylated polystyrene nanoparticles were verified to be of the size described by the manufacturer's specification and they were shown to exhibit a negative zeta potential (Table 1). The polystyrene particle's zeta potential reduced as $\mathrm{pH}$ of the dispersion medium became more acidic (Table 1).

Table 1. Nanomaterial characteristics and barrier diffusion rates. Data represents the mean \pm standard deviation $(n=3)$. Symbols indicate a statistical difference when comparing the results across the groups which differed in *particle size, ${ }^{\#} \mathrm{pH}$ and ${ }^{\Delta}$ surface chemistry $(\mathrm{p}<0.05)$. PS denotes polystyrene, LNC lipid nanocapsules and DLNC distended lipid nanocapsules (diameter denotes the non-distended dimension, note the distended size was 75 $\mathrm{nm}$ at $100 \mathrm{~min}$ ). PGM represents porcine gastric mucin and CFM cystic fibrosis mucus. $\mathrm{D}_{\mathrm{w}}$ is the nanomaterial diffusion in water and $\mathrm{D}_{\mathrm{m}}$ is the diffusion through the barrier. ND refers to no diffusion detected in the 60 min time period of measurement.

\begin{tabular}{|c|c|c|c|c|c|c|}
\hline Test System & $\begin{array}{c}\text { Diameter } \\
(\mathrm{nm})\end{array}$ & $\begin{array}{c}\xi- \\
\text { potential, } \\
(\mathrm{mV})\end{array}$ & $\begin{array}{c}\text { Diffusivity in } \\
\text { PGM, (x 10 } \\
\left.{ }^{10}\right)\left(\mathrm{cm}^{2} \mathrm{~s}^{-1}\right)\end{array}$ & $\begin{array}{l}D_{w} / D_{m} \\
\text { PGM }\end{array}$ & $\begin{array}{c}\text { Diffusivity in } \\
\text { CFM, (x 10 } \\
\left.{ }^{10}\right)\left(\mathrm{cm}^{2} \mathrm{~s}^{-1}\right)\end{array}$ & $\begin{array}{c}D_{\mathrm{w}} / \mathbf{D}_{\mathrm{m}} \\
\text { CFM }\end{array}$ \\
\hline PS50 pH 8.5 & $53 \pm 1$ & $-35.9 \pm 0.8$ & $9.87 \pm 0.61$ & 132.9 & $1.14 \pm 0.20$ & 1150.7 \\
\hline PS200 pH 8.5 & $187 \pm 2$ & $-48.4 \pm 1.4$ & $19.93 \pm 5.47$ & 16.5 & ND & - \\
\hline PS750 pH 8.5 & $778 \pm 3$ & $-61.5 \pm 1.0$ & $6.67 \pm 1.28^{*}$ & 13.1 & ND & - \\
\hline PS200 pH 6.5 & $185 \pm 2$ & $-41.8 \pm 3.9$ & $9.54 \pm 3.26$ & 34.4 & ND & - \\
\hline PS200 pH 2.5 & $183 \pm 2$ & $-33.2 \pm 1.1$ & $2.98 \pm 0.74 \#$ & 11.0 & ND & - \\
\hline LNC50 pH 8.5 & $52 \pm 2$ & $-3.5 \pm 0.6$ & $30.08 \pm 2.49 \Delta$ & 43.6 & $28.99 \pm 3.28 \Delta$ & 45.3 \\
\hline $\mathrm{DLNC}^{\circ} 0^{\mathrm{a}} \mathrm{pH} 8.5$ & $52 \pm 2$ & $-2.4 \pm 1.0$ & $27.10 \pm 3.84 \Delta$ & 48.4 & $29.51 \pm 2.13 \Delta$ & 44.5 \\
\hline
\end{tabular}

The $200 \mathrm{~nm}$ polystyrene particles moved across the PGM barrier twice as fast as the

$50 \mathrm{~nm}$ particles and three times faster than the $750 \mathrm{~nm}$ particles $(\mathrm{p}<0.05$; Fig.3A). The fastest rate of diffusion for the $200 \mathrm{~nm}$ PS nanoparticles was observed at $\mathrm{pH} 8.5$, which was 2- and 7-fold faster compared to $\mathrm{pH} 6.5$ and $\mathrm{pH} 2.5$, respectively ( $<<0.05$; Fig.3B, Table 1). The PGM had the capability of restricting the polystyrene nanoparticle movement such that diffusion was up to 133 times slower than the calculated diffusion in water (Table 1). 


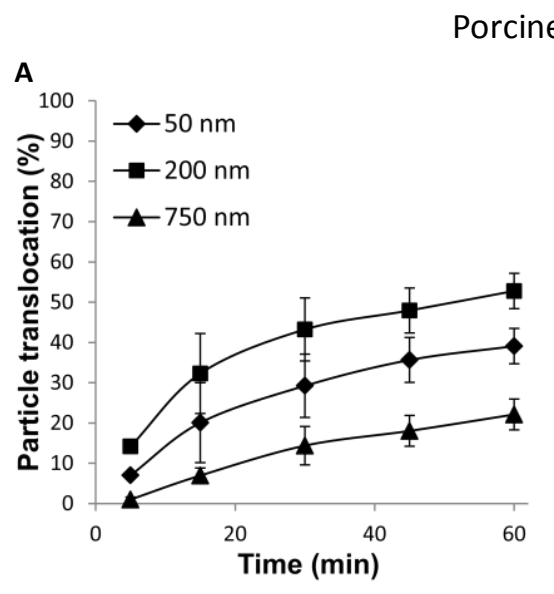

Cystic Fibrosis Mucus
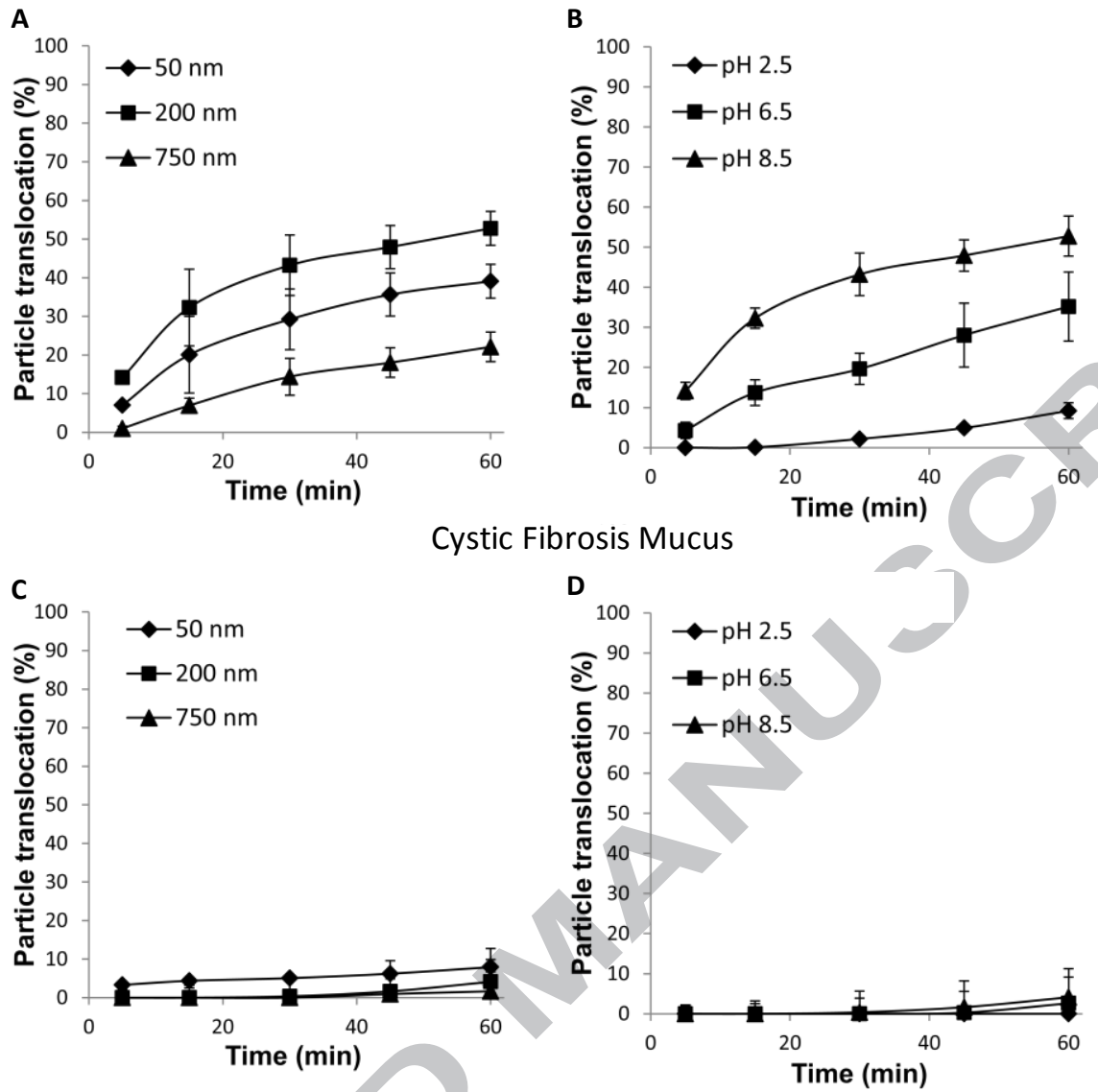

Fig.3.(A) The translocation of 50,200 and $750 \mathrm{~nm}$ polystyrene nanoparticles (pH 8.5) across porcine gastric mucin (PGM) (B) The translocation of $200 \mathrm{~nm}$ polystyrene nanoparticles at $\mathrm{pH} 2.5,6.5$ or 8.5 across PGM (C) The translocation of 50, 200 and $750 \mathrm{~nm}$ polystyrene nanoparticles ( $\mathrm{pH} 8.5$ ) across cell line derived cystic fibrosis mucus (D) The translocation of $200 \mathrm{~nm}$ polystyrene nanoparticles at $\mathrm{pH} 2.5,6.5$ or 8.5 across cystic fibrosis mucus. Data represent mean \pm standard deviation $(\mathrm{n}=3)$.

The LNCs were $50 \mathrm{~nm}$ in diameter with a small negative zeta potential (Table 1). Their diffusion rate across the PGM was 3 times faster than the equivalent-sized negatively charged polystyrene particles $(\mathrm{p}<0.05$; Fig. 3 ). When the LNC distension process was triggered (size increase is reported in supplementary data Fig S6, this data matched that reported in previous work $^{19}$ ), the diffusion rate across the PGM was equivalent to non-distended particles ( $\mathrm{p}>$ 0.05), indicating that surfactant-induced particle distension had little effect on translocation in this model (Fig.3B). The effect of the surfactant on the barrier structure could not be determined, but if the surfactant broke down the barrier structure it may have been 
counterbalancing the size change experienced during nanomaterial size distension, which would be expected to slow the particle diffusion down. The diffusion of the LNCs was approximately 45 times slower in the PGM than in water (Table 1). Over 60 min almost all of the LNCs that were applied to the apical surface of the PGM barrier passed through it and this demonstrated that the SLN translocation was more extensive compared to the polystyrene particles (maximum translocation approximately 50\%) over the 60 min time period.
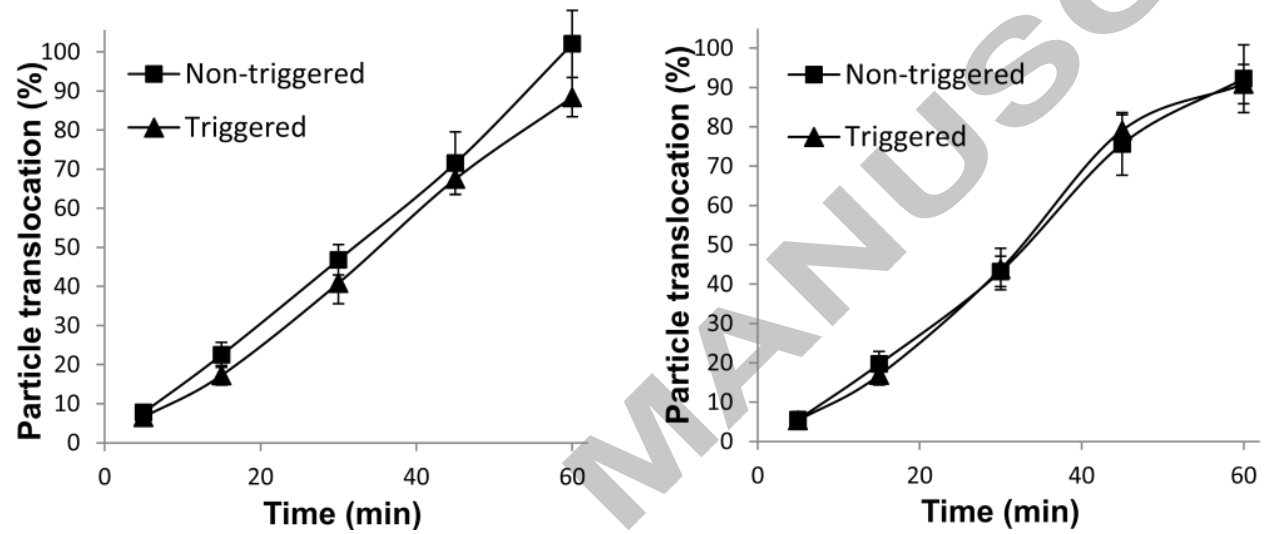

Fig.4. The translocation of $50 \mathrm{~nm}$ lipid nanocapsules (LNCs) upon triggered distension compared to non-triggered $50 \mathrm{~nm}$ LNCs through a $35 \mu \mathrm{m}$ thick (A) purified porcine gastric mucin (PGM) gel at $\mathrm{pH}$ 8.5. and (B) cell-line derived cystic fibrosis mucus gel at $\mathrm{pH}$ 8.5. Data represent mean \pm standard deviation $(n=3)$.

\subsection{Nanomaterial Translocation across CFM}

The translocation of the polystyrene nanoparticles across the static CFM barrier was much slower compared to the translocation across PGM for all the polystyrene particles (Table 1; Fig. 3C and 3D). Only the $50 \mathrm{~nm}$ particles, when applied in a buffer at $\mathrm{pH} 8.5$, generated a particle translocation that was significantly different to zero at the 60 min time point and therefore the other systems were considered to be effectively immobile in the CFM over this time frame. Further, experiments were not conducted to confirm if the nanomaterials were immobile or simply diffusing very slowly because even if they were 
diffusing very slowly it was thought unlikely that such particles would be investigated as drug delivery vectors. The magnitude of retardation of the $50 \mathrm{~nm}$ polystyrene particle diffusion in the CFM compared to the PGM was approximately 10 -fold ( $\mathrm{p}<0.05$; Table 1$)$.

The LNCs exhibited a 30-fold higher translocation rate compared to similar sized polystyrene particles $(\mathrm{p}<0.05)$, irrespective of the distension process, in CFM (Fig,4A and B). Unlike the polystyrene particles, LNC diffusivity was not slowed down in the CFM compared to PGM. Almost all the LNCs passed the barrier in the $60 \mathrm{~min}$ time frame, which suggested the particle diffusion was extensive and unidirectional through CFM ( $\mathrm{p}>0.05)$.

As the diffusion of all the nanomaterials was detectable in PGM only this barrier was used in the particle tracking studies in order to understand how the hydrodynamic and steric interactions influenced the nanomaterial diffusion in the gel.

\subsection{Nanomaterial Tracking in PGM}

The mean particle diffusion rates were up to thirty times greater in PGM gels mobilised in the flow through cell compared to the Transwell diffusion studies according the nanomaterial tracking measurements (Fig 5). In the mobile PGM system even though it appeared that the $50 \mathrm{~nm}$ sized polystyrene particles diffused at a faster rate than the $200 \mathrm{~nm}$ and $750 \mathrm{~nm}$ particles the error associated with the measurements resulted in no statistically significant differences between the particle of different sizes ( $p>0.05$, Fig.5). The distended LNCs did diffuse more slowly $\left(3.97 \pm 1.38 \times 10^{-8} \mathrm{~cm}^{2} / \mathrm{s}, \mathrm{p}<0.05\right)$ compared to the nondistended materials $\left(4.94 \pm 0.04 \times 10^{-8} \mathrm{~cm}^{2} / \mathrm{s}\right.$, Fig.5)). The LNC data suggested that particle size (the distended materials swelled up to a size of $250 \mathrm{~nm}$ ) was important in the nanomaterial diffusion profile in the absence of steric effects for the soft lipid materials. 


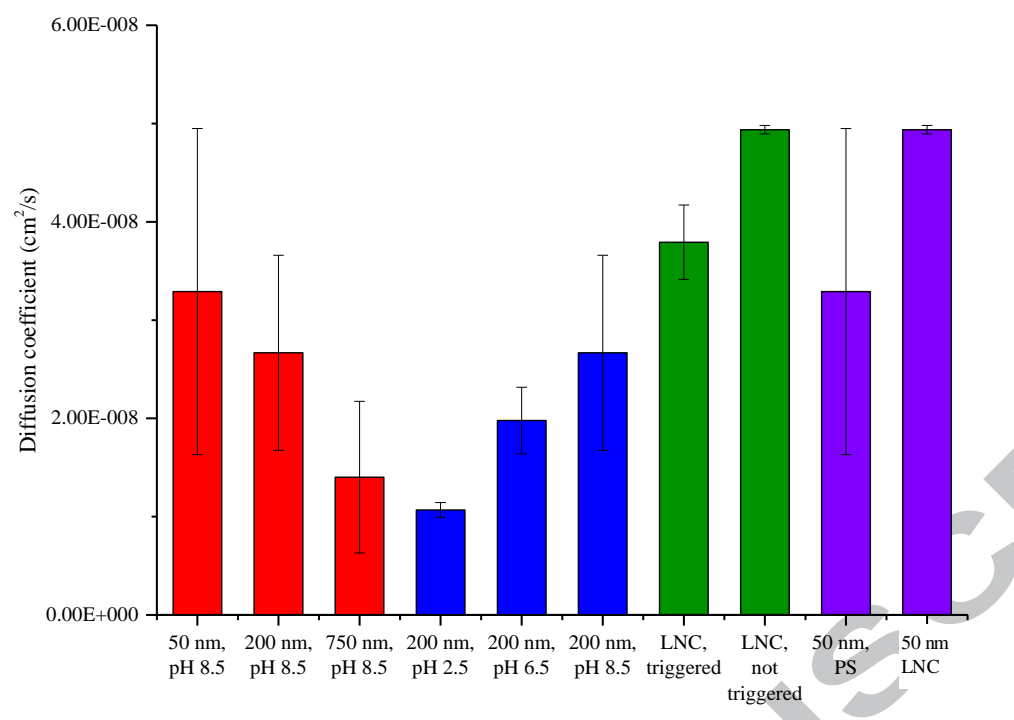

Fig.5. The diffusion coefficients of polystyrene and lipid nanoparticles in porcine gastric mucin (PGM) displaying the effect of polystyrene particle size (red), effect of media $\mathrm{pH}$ on polystyrene $200 \mathrm{~nm}$ nanoparticles (blue), distension triggered and non-triggered lipid nanocapsules (LNCs) (green) and nanoparticle composition (purple). Data represent the means \pm standard deviation, where $n=3$.

It was notable that there was a wide range of particle diffusion coefficients measured for each polystyrene sample, i.e., the measurement polydispersity was relatively high in comparison to that obtained in polymer gels in previous work, ${ }^{13}$ but as the nanomaterial particle size increased the diffusion coefficient profile appeared to become more monodisperse (see Supplementary material Fig S7 for the diffusion distribution data). Changing the $\mathrm{pH}$ of the mobile PGM gel showed very little change in measurement polydispersity, but it did have an influence on the diffusion of the nanomaterials through the mobile PGM gel. The diffusion coefficient at $\mathrm{pH} 2.5$ was significantly different $(\mathrm{p}<0.05)$ to $\mathrm{pH} 6.5$ and $\mathrm{pH} 8.5$, but there was no significant difference between the $\mathrm{pH} 6.5$ and 8.5 groups of data (Fig S7 and Fig.4, pH $2.5-1.07 \pm 0.07 ; \mathrm{pH} 6.5-1.98 \pm 0.34 ; \mathrm{pH} 8.5-2.67 \pm 0.99 \times 10^{-}$ $\left.{ }^{8} \mathrm{~cm}^{2} / \mathrm{s}\right)$.

There appeared to be two important differences between the comparisons of the particle diffusion speeds in the translocation studies and the particle tracking studies. Firstly, the 50 
$\mathrm{nm}$ LNC diffusion was very similar to the $50 \mathrm{~nm}$ polystyrene nanomaterial diffusion in the particle tracking experiments $\left(4.94 \pm 0.04 \times 10^{-8} \mathrm{~cm}^{2} / \mathrm{s}\right.$, compared to $3.29 \pm 165 \times 10^{-8} \mathrm{~cm}^{2} / \mathrm{s}$, respectively, no statistical significant difference observed). Secondly, in the particle tracking measurements, triggering the distension of the LNC reduced their diffusion speed and this was not observed in the translocation studies. These differences were a consequence of disrupting the barrier structure and this implied that it was the modification of LNC's steric interactions with the barrier that underpinned their ability to penetrate the barrier faster than the polystyrene materials.

\section{Discussion}

Measuring particle diffusion using the translocation assay, which employed $35 \mu \mathrm{m}$ thick PGM and CFM barriers mounted in Transwell diffusion cells, provided evidence to suggest that the -ve polystyrene nanoparticles were mucuoadhesive. ${ }^{32}$ In this work the mucoadhesion of the polystyrene particles resulted in the $50 \mathrm{~nm}$ sized materials passing through the PGM more slowly compared to the $200 \mathrm{~nm}$ sized materials. This type of diffusion data has previously been reported and it has been suggested that slower translocation of small nanomaterials across mucin rich barriers is a consequence of the materials taking a more tortuous diffusion path, i.e., the particles experience more extensive steric interactions with the mucin gel. ${ }^{14}$ The diffusion measurements in a mobile PGM bed supported this theory, because when the mucin structure was disrupted and the steric interactions were supressed there was no statistical difference between the diffusion of the polystyrene particles of different sizes. However, the large error associated with the diffusion measurements in the mobile PGM beds and the broad diffusion ranges of the particles suggested that there were also significant hydrodynamic interactions between the mucin and the particles even when the steric interactions were supressed by perturbing the PGM structure. ${ }^{34}$ 
Reducing the PGM pH appeared to slow the diffusion of the nanomaterials, both in the Transwell and the particle tracking diffusion measurements. This could have been a consequence of the change in ionisation of the carboxyl and sulphated groups present on the particle surface, which were fully ionised between $\mathrm{pH}$ 5-9 (Manufacturer's data), but only partially ionised $\mathrm{pH}$ 2. However, because a greater $\mathrm{pH}$ induced reduction in diffusion was observed in the translocation experiments compared to the particle tracking experiments this again suggested that the steric interactions had a more profound impact on the particle diffusion compared to hydrodynamic interactions, hence the effects of $\mathrm{pH}$ on the mucus barrier were perhaps more consequential. A tighter barrier is produced by the mucin at acidic $\mathrm{pHs}$ and this probably was the cause of the enhanced the diffusion restriction observed at $\mathrm{pH}$ 2 compared to $\mathrm{pH} 8.5{ }^{32}$ At low $\mathrm{pHs}$ it was also more likely that the carboxylated polystyrene nanoparticles, which do possess a hydrophobic surface ${ }^{33}$, showed stronger interactions with mucus mucin through hydrophobic interactions. ${ }^{35}$

In the CFM the $50 \mathrm{~nm}$ polystyrene materials diffused more rapidly compared to the 200 nm materials. Therefore, it was unlikely that the same mechanism of diffusion restriction was acting on the polystyrene materials in both the PGM and CFM. The principle differences between CFM and PGM were that PGM contained more mucin ${ }^{36}$ whilst CFM contained a larger amount of extracellular material. ${ }^{37}$ The extracellular material in CFM would effectively fill the mesh spaces in the gel and this could increase the steric interactions between the gel and the nanomaterials and modify the hydrodynamic interactions by shielding the mucin fibres from interacting with the particles. In addition, is it likely that the mucins from the cell culture system are of gel forming quality and therefore my retain more of the domains that can interact with itself (smaller pores) and the nanomaterials. Unfortunately, direct evidence of which of the two interaction types, steric or hydrodynamic, were most influential in the 
CFM mucus could not be gathered in this work because of the low mobility of the particles in the CFM during the diffusion cell measurements and the large amount of CFM required for the particle-tracking measurements using the commercial equipment.

The similar translocation speed of the $50 \mathrm{~nm}$ PEG coated LNCs across the mucin rich barriers irrespective of the barrier composition or the LNC distension process demonstrated that the LNC had very limited interactions with the barrier. These findings were in agreement with previously published work that has shown that both $50 \mathrm{~nm}$ and $300 \mathrm{~nm}$ PEG coated materials penetrated respiratory mucus to extend nanoparticle residence at the surface of the epithelium. ${ }^{31}$ It appeared that the steric interactions of the LNCs were modified more than the hydrodynamic interactions in PGM when comparing the LNCs to the $50 \mathrm{~nm}$ polystyrene particles which was in agreement with the previous observations made in this work.

Both the LNC and the polystyrene nanomaterials appeared to show unidirectional movement through the mucin rich barriers in the translocation studies, albeit over different timescales (LNC 100\% diffusion in $60 \mathrm{~min}$ and polystyrene in $500 \mathrm{~min}$ ). Leon et al., (2013), who used a novel microchannel diffusion method to understand both the material diffusion and the partitioning into the mucus barrier, suggested that the Donnan transport process, which drives uni-directional diffusion, played a role in the transport of nanomaterials across mucus barriers. ${ }^{40}$ In the current study there were two major gradients in the mucus translocation experiments, the particle concentration gradient and the electrolyte gradient, both were passive transport processes. The electrolyte gradient was established as a consequence of applying $1 \mu \mathrm{L}$ of $50 \mathrm{mM}$ tris buffer to the apical surface of the mucus and $600 \mu \mathrm{L}$ of tris buffer to the basolateral side. When more Tris was applied to the apical surface of the mucin rich barrier the rate of particle diffusion was suppressed. The barrier swelling did not change upon addition of the different volumes of Tris and so either the buffer 
transport was involved in the particle diffusion process or the buffer was shielding the electrostatic interactions of the mucin. Previous work has shown that charge shielding of the mucin electrostatic interactions with nanomaterials by the addition of high electrolyte concentrations does not decrease, but rather increases the diffusion of charged nanomaterials ${ }^{41}$ and so the data reported in the translocation experiments were thought to be most likely influenced Donnan phenomena ${ }^{42}$. As the electrolyte effects were only tested with the polystyrene materials in the current study further work is needed to understand how electrolyte gradients could function to modify the translocation of soft nanomaterials exhibiting different properties across mucus barriers.

The thirty fold increase in nanomaterial diffusion coefficients when shear was applied to the PGM compared to those calculated for the same materials in the static gel suggested that the mucin structural integrity was an important factor in its ability to hinder nanoparticle diffusion. Previous work has noted that the most hydrophobic domains of the mucus are at the points of mucin cross links and in this study the shear imparted on the mucin rich barrier during flow through would most probably break these regions down. ${ }^{38}$ The notion that the mucus cross links were important in hydrophobic interactions with the nanomaterials fits in well with the previous suggestion that the polystyrene materials were interacting the mucus through hydrophobic interaction, which were more likely to occur at the mucus cross-link points. In addition, it provides an explanation as to why the LNCs showed a similar diffusion rate in the nanoparticle tracking measurements compared to the polystyrene nanomaterials, whereas in the Transwell translocation experiments the LNCs diffused significantly faster. If hydrophobic interactions were important and they were at the cross linking points of the mucin then the PEG protection on the LNCs would be most effective when the gel was intact. This close relationship between hydrodynamic and structural interaction forces with the 
mucus structure may be the reason why it is not easy to experimentally separate and measure these two types of interactions in different types of mucus systems and suggests that theoretical and experimental procedures need to be designed to probe these interactions more carefully in order to gain a better understanding of nanomaterials penetration through mucus gels.

\section{CONCLUSIONS}

The calculation of diffusion coefficients using both particle translocation and particle tracking measurements showed that the PEG coated, soft, distensible lipid $50 \mathrm{~nm}$ nanoparticles efficiently penetrated both PGM and CFM. A comparison of the data from the two diffusion measurement techniques suggested that the efficient translocation of the LNCs was a consequence of diminished hydrophobic interactions with the barrier compared to solid polystyrene materials of an equivalent size. The PEG coating of the LNC was equally effective in protecting the materials from mucin interactions in both PGM and CFM and this suggested that the hydrodynamic and steric interactions were inherently linked to the mucus structure. These findings can guide the design of nanomaterials for applications such as gene delivery where the active needs to cross the mucus barrier to reach epithelial target cells. ${ }^{43}$ Intriguingly, the data presented in the study also suggested that electrical gradients established during diffusion of surface charged nanomaterials through mucus barriers may facilitate their unidirectional transport and this provides an interesting hypothesis to peruse in further work. In addition, an immediate areas for future work is the delivery of the triggered release LNCs reported herein to the lung as a respirable aerosol. This could be achieved using a dual chamber device that allows the physical separation of the trigger and the LNCs, e.g. the prototype the 'Duohaler ${ }^{\circledR}$ ' available from Vectura Ltd, which would allow mixing only upon dose actuation. 


\section{SUPPORTING INFORMATION}

The Supporting Information is available in Supplementary data (PDF). 


\section{REFERENCES}

1. Chen, H.; Woods, A.; Forbes, B.; Jones, S. Controlled drug release from lungtargeted nanocarriers via chemically mediated shell permeabilisation. Int. J. Pharm. 2016, $511,(2), 1033-1041$.

2. Mura, S.; Nicolas, J.; Couvreur, P. Stimuli-responsive nanocarriers for drug delivery. Nature Mater. 2013, 12, (11), 991.

3. Ganta, S.; Devalapally, H.; Shahiwala, A.; Amiji, M. A review of stimuli-responsive nanocarriers for drug and gene delivery. J. Control. Release. 2008, 126, (3), 187-204.

4. Cone, R. A. Barrier properties of mucus. Adv. Drug Delivery Rev. 2009, 61, (2), 7585.

5. Wang, Y.-Y.; Lai, S. K.; So, C.; Schneider, C.; Cone, R.; Hanes, J. Mucoadhesive nanoparticles may disrupt the protective human mucus barrier by altering its microstructure. PLoS One 2011, 6, (6), e21547.

6. Tagalakis, A. D.; Maeshima, R.; Yu-Wai-Man, C.; Meng, J.; Syed, F.; Wu, L. P.; Aldossary, A. M.; McCarthy, D.; Moghimi, S. M.; Hart, S. L. Peptide and nucleic aciddirected self-assembly of cationic nanovehicles through giant unilamellar vesicle modification: Targetable nanocomplexes for in vivo nucleic acid delivery. Acta Biomater. 2017, 51, 351-362.

7. Sanders, N. N.; DE SMEDT, S. C.; Van Rompaey, E.; Simoens, P.; De Baets, F.; Demeester, J. Cystic fibrosis sputum: a barrier to the transport of nanospheres. Am. J. Respir. Crit. Care Med. 2000, 162, (5), 1905-1911.

8. Schuster, B. S.; Suk, J. S.; Woodworth, G. F.; Hanes, J. Nanoparticle diffusion in respiratory mucus from humans without lung disease. Biomaterials 2013, 34, (13), 34393446.

9. Roblegg, E.; Fröhlich, E.; Meindl, C.; Teubl, B.; Zaversky, M.; Zimmer, A. Evaluation of a physiological in vitro system to study the transport of nanoparticles through the buccal mucosa. Nanotoxicology 2012, 6, (4), 399-413.

10. Lai, S. K.; Suk, J. S.; Pace, A.; Wang, Y.-Y.; Yang, M.; Mert, O.; Chen, J.; Kim, J.; Hanes, J. Drug carrier nanoparticles that penetrate human chronic rhinosinusitis mucus. Biomaterials 2011, 32, (26), 6285-6290.

11. Crater, J. S.; Carrier, R. L. Barrier properties of gastrointestinal mucus to nanoparticle transport. Macromo Biosci. 2010, 10, (12), 1473-1483.

12. Shan, W.; Zhu, X.; Liu, M.; Li, L.; Zhong, J.; Sun, W.; Zhang, Z.; Huang, Y. Overcoming the Diffusion barrier of mucus and absorption barrier of epithelium by selfassembled nanoparticles for oral delivery of insulin. ACS Nano 2015, 9, (3), 2345-2356. 
13. Mun, E. A.; Hannell, C.; Rogers, S. E.; Hole, P.; Williams, A. C.; Khutoryanskiy, V. V. On the Role of Specific Interactions in the Diffusion of Nanoparticles in Aqueous Polymer Solutions. Langmuir 2014, 30, (1), 308-317.

14. Lai, S. K.; O'Hanlon, D. E.; Harrold, S.; Man, S. T.; Wang, Y.-Y.; Cone, R.; Hanes, J. Rapid transport of large polymeric nanoparticles in fresh undiluted human mucus. Proc. Natl. Acad. Sci. 2007, 104, (5), 1482-1487.

15. Lieleg, O.; Ribbeck, K. Biological hydrogels as selective diffusion barriers. Trends Cell Biol. 21, (9), 543-551.

16. Squires, T. M.; Mason, T. G. Fluid mechanics of microrheology. Annu. Rev.Fluid Mech. 2010, 42.

17. Phillips, R.; Deen, W.; Brady, J. Hindered transport of spherical macromolecules in fibrous membranes and gels. AIChE journal 1989, 35, (11), 1761-1769.

18. Spero, R. C.; Sircar, R. K.; Schubert, R.; Taylor, R. M.; Wolberg, A. S.; Superfine, R. Nanoparticle diffusion measures bulk clot permeability. Biophys. J. 2011, 101, (4), 943-950.

19. Chana, J.; Forbes, B.; Jones, S. A. Triggered-release nanocapsules for drug delivery to the lungs. Nanomedicine: Nanotechnology, Biology and Medicine 2015, 11, (1), 89-97.

20. Celli, J.; Gregor, B.; Turner, B.; Afdhal, N. H.; Bansil, R.; Erramilli, S. Viscoelastic properties and dynamics of porcine gastric mucin. Biomacromolecules 2005, 6, (3), 13291333.

21. Gendler, S. J.; Spicer, A. Epithelial mucin genes. Annu. Rev. Physiol. 1995, 57, (1), 607-634.

22. Heurtault, B.; Saulnier, P.; Pech, B.; Proust, J.-E.; Benoit, J.-P. A novel phase inversion-based process for the preparation of lipid nanocarriers. Pharm. Res. 2002, 19, (6), 875-880.

23. Diamant, H. Hydrodynamic interaction in confined geometries. J. Phys. Soc. Jpn 2009, 78, (4), 041002.

24. Wagner T, Kroll A, Haramagatti CR, Lipinski H-G, Wiemann M. Classification and Segmentation of Nanoparticle Diffusion Trajectories in Cellular Micro Environments. PLoS ONE. 2017 12(1): e0170165.

25. Lai, S. K.; Wang, Y.-Y.; Wirtz, D.; Hanes, J. Micro-and macrorheology of mucus. Adv. Drug Delivery Rev. 2009, 61, (2), 86-100.

26. Dawson, M.; Wirtz, D.; Hanes, J. Enhanced viscoelasticity of human cystic fibrotic sputum correlates with increasing microheterogeneity in particle transport. J. Biol. Chem. 2003, 278, (50), 50393-50401. 
27. App, E. M.; Zayas, J. G.; King, M. Rheology of mucus and transepithelial potential difference: small airways versus trachea. Eur. Respir. J. 1993, 6, (1), 67-75.

28. Wesley, A. W.; Forstner, J. F.; Forstner, G. G. Structure of intestinal-mucus glycoprotein from human post-mortem or surgical tissue: Inferences from correlation analyses of sugar and sulfate composition of individual mucins. Carbohydr. Res. 1983, 115, 151-163.

29. Wesley, A.; Forstner, J.; Qureshi, R.; Mantle, M.; Forstner, G. Human Intestinal Mucin in Cystic Fibrosis. Pediatr Res 1983, 17, (1), 65-69.

30. Mühlfeld, C.; Rothen-Rutishauser, B.; Blank, F.; Vanhecke, D.; Ochs, M.; Gehr, P. Interactions of nanoparticles with pulmonary structures and cellular responses. Am. J. Physiol.-Lung Cellular and Molecular Physiology 2008, 294, (5), L817-L829.

31. Schneider, C. S.; Xu, Q.; Boylan, N. J.; Chisholm, J.; Tang, B. C.; Schuster, B. S.; Henning, A.; Ensign, L. M.; Lee, E.; Adstamongkonkul, P. Nanoparticles that do not adhere to mucus provide uniform and long-lasting drug delivery to airways following inhalation. Science Advances 2017, 3, (4), e1601556.

32. Lieleg, O.; Vladescu, I.; Ribbeck, K. Characterization of particle translocation through mucin hydrogels. Biophys. J. 2010, 98, (9), 1782-1789.

33. Jones, M.-C.; Jones, S. A.; Riffo-Vasquez, Y.; Spina, D.; Hoffman, E.; Morgan, A.; Patel, A.; Page, C.; Forbes, B.; Dailey, L. A. Quantitative assessment of nanoparticle surface hydrophobicity and its influence on pulmonary biocompatibility. J. Control. Release. 2014, 183, 94-104.

34. Ando, T.; Skolnick, J. Crowding and hydrodynamic interactions likely dominate in vivo macromolecular motion. Proc. Natl. Acad. Sci. 2010, 107, (43), 18457-18462.

35. Norris, D. A.; Sinko, P. J. Effect of size, surface charge, and hydrophobicity on the translocation of polystyrene microspheres through gastrointestinal mucin. J. Appl. Polym. Sci. 1997, 63, (11), 1481-1492.

36. Hill, D. B.; Vasquez, P. A.; Mellnik, J.; Mckinley, S. A.; Vose, A.; Mu, F.; Henderson, A. G.; Donaldson, S. H.; Alexis, N. E.; Boucher, R. C. A biophysical basis for mucus solids concentration as a candidate biomarker for airways disease. Plos One 2014, 9, (2), e87681.

37. Rubin, B. K. Mucus structure and properties in cystic fibrosis. Paediatr. Respir. Rev. 2007, 8, (1), 4-7.

38. Bansil, R.; Turner, B. S. Mucin structure, aggregation, physiological functions and biomedical applications. Curr. Opin. Colloid Interface Sci. 2006, 11, (2-3), 164-170.

39. Wang, Y. Y.; Lai, S. K.; Suk, J. S.; Pace, A.; Cone, R.; Hanes, J. Addressing the PEG mucoadhesivity paradox to engineer nanoparticles that "slip" through the human mucus barrier. Angew.Chem. Int. Edit. 2008, 47, (50), 9726-9729. 
40. Li, L. D.; Crouzier, T.; Sarkar, A.; Dunphy, L.; Han, J.; Ribbeck, K. Spatial configuration and composition of charge modulates transport into a mucin hydrogel barrier. Biophys. J. 2013, 105, (6), 1357-1365.

41. Zhang, X.; Hansing, J.; Netz, R. R.; DeRouchey, J. E. Particle transport through hydrogels is charge asymmetric. Biophys. J. 2015, 108, (3), 530-539.

42. Tam, P. Y.; Verdugo, P. Control of mucus hydration as a Donnan equilibrium process. Nature 1981, 292, (5821), 340-342.

43. Tagalakis AD, Munye MM, Ivanova R, et al Effective silencing of ENaC by siRNA delivered with epithelial-targeted nanocomplexes in human cystic fibrosis cells and in mouse lung Thorax Published Online First: 10 May 2018. doi: 10.1136/thoraxjnl-2017-210670

44. H. Nordman, J.R. Davies, A. Herrmann, N.G. Karlsson, G.C. Hansson, I. Carlstedt Biochem. J., 326 (1997), pp. 903-910 


\section{GRAPHICAL ABSTRACT}
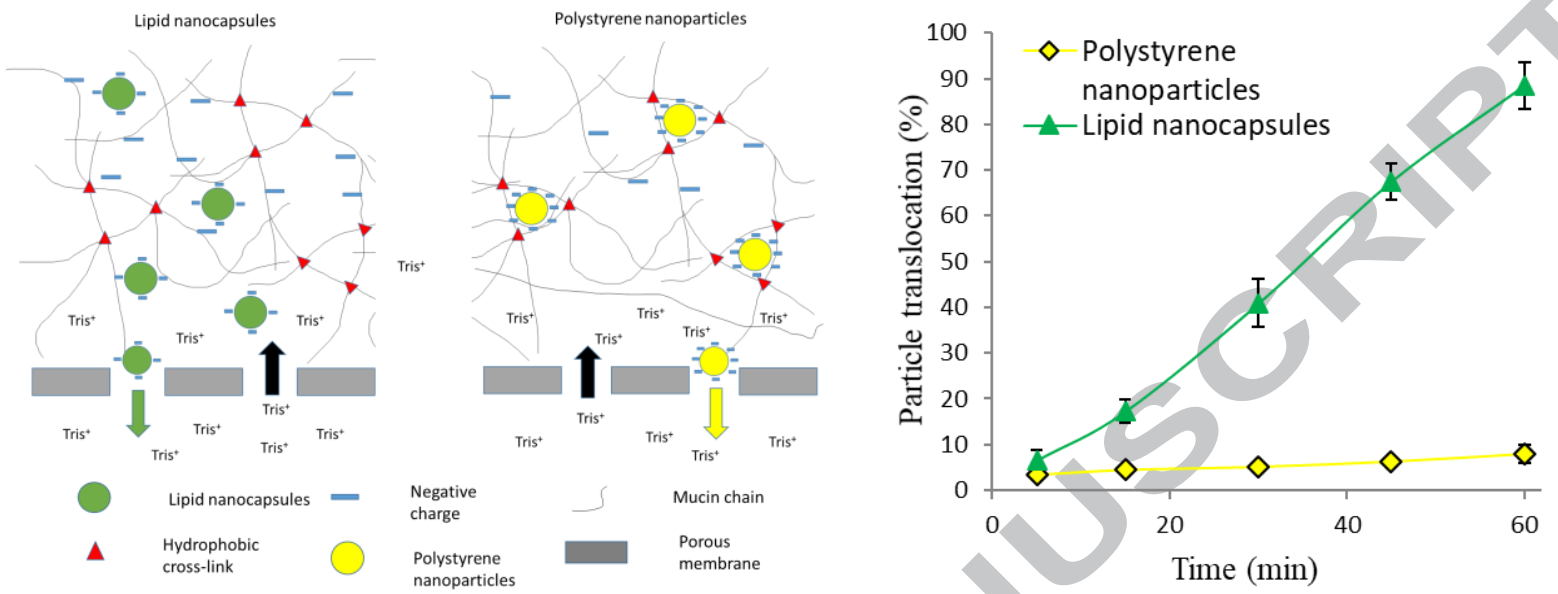